\title{
Electrocardiographic diagnosis of the left ventricular hypertrophy in patients with left bundle branch block: Is it necessary to verify old criteria?
}

\author{
Rafał Baranowski, Łukasz Małek, Dominika Prokopowicz, \\ Mateusz Śpiewak, Jolanta Miśko \\ Institute of Cardiology, Warsaw, Poland
}

\begin{abstract}
Background: The diagnosis of left ventricular hypertrophy (LVH) in the presence of the left bundle branch block (LBBB) is difficult. In our study we compared commonly used ECG criteria with left ventricular mass index (LVMI) calculated during cardiac magnetic resonance imaging (CMRI) to verify their clinical value or the need to recalibrate.

Methods: CMRI and ECG data of 36 patients were included in this study. Based on the ECG measures we used in our study selected ECG criteria for LVH detection in cases with LBBB: $Q R S$ duration, amplitude of $S$ waves in V1,V2,V3; R waves in $a V L, V 5, V 6$ and combinations of amplitudes. LVH was defined as LVMI (CMRI) exceeding reference values.
\end{abstract}

Results: LVH was diagnosed in 17 (47\%) patients (6 women and 11 men). Following ECG parameters correlated the most prominently with LVMI $-R V 5: r=0.5(p=0.002), R V 6$ : $r=0.61(p=0.0001), S V 1+R V 5,6: r=0.64(p=0.001), R a V L+S V 3: r=0.5(p=0.002)$, SV2+RV5, $6: r=0.71(p=0.0001), S V 2,3+R V 5,6: r=0.75(p=0.0001)$. Based on the results of ROC analysis we proposed new cut points for LVH parameters. The highest diagnostic accuracy achieved $S 2+S V 3>6 m V, S V 2, V 3+R V 5, V 6>4 m V, R a V L+S V 3>3.5 m V(86-89 \%)$.

Conclusions: In patients with $L B B B$ the ECG parameters based on the amplitude of $S$ wave in V2 or V3 and $R$ wave in the leads aVL, V5, V6 have the highest clinical value in the prediction of the LVH determined by CMRI. It was necessary to recalibrate these parameters and it is needed to verify them in larger number of LBBB patients. (Cardiol J 2012; 19, 6: 591-596)

Key words: ECG, left bundle branch block, left ventricular hypertrophy, cardiac magnetic resonance imaging

\section{Introduction}

The diagnosis of left ventricular hypertrophy $(\mathrm{LVH})$ in the presence of the left bundle branch block (LBBB) is difficult or even in opinion of some authors impossible [1]. Abnormal ventricular conduction usually changes (in either direction) the QRS amplitude [1]. Classical parameters that are used for QRS $<120 \mathrm{~ms}$ are generally not suitable.
We also know that the important percentage of patients with LBBB have hypertrophied or dilated hearts [2]. Cardiac magnetic resonance imaging (CMRI) is currently used as a gold standard technique for LV size measurements [3]. It is more precise in LV measurements than echo [4-6]. To our knowledge ECG criteria for LVH diagnosis in patients with LBBB have not been verified with CMRI results. In our study we compared commonly used

Address for correspondence: Rafał Baranowski, MD, PhD, Associate Professor, Institute of Cardiology, ul. Alpejska 42, 04-628 Warszawa, Poland, tel/fax: +48 2234344 09, e-mail: rbaranowski@ikard.pl 
ECG criteria with left ventricular mass index (LVMI) calculated during CMRI to verify their clinical value or the need to update.

\section{Methods}

From the patients that had CMRI of the heart in Institute of Cardiology during 2009 and till 30.08.2010 we selected cases with LBBB in the ECG. The examined group finally consisted of 36 adults, mean age 56 (33-80) years, 21 males and 15 females. Fifteen patients had dilated cardiomyopathy, 9 ischemic cardiomyopathy, 4 hypertrophic cardiomyopathy and in 8 patients other pathology was diagnosed.

The study was approved by the local bioethical committee.

\section{Electrocardiography}

All of the ECG were recorded digitally with Spacelabs Syphonet/Sentinel workstations. ECG waves and intervals (amplitude and duration) were measured by one observer (D.P.) using digital calipers and verified by the other (R.B.). LBBB was diagnosed using the most recent published criteria [7]:

- QRS duration $\geq 120 \mathrm{~ms}$;

- board, notched or slurred $\mathrm{R}$ wave in leads I, aVL, V5 and V6;

- absent q waves in leads I, V5, V6;

- $\mathrm{R}$ peak time $>60 \mathrm{~ms}$ in leads V5 and V6 but normal in V1, V2, V3;

- ST and T waves usually opposite in direction to QRS.

The separation between LBBB and QRS prolongation due to $\mathrm{LVH}$ is a well known problem in electrocardiology. For this reason we include the cases with QRS > 130 ms.

In our study, we used the following ECG measurements: QRS duration (max in 12 leads), $P$ wave duration in lead II (in patients with sinus rhythm), $\mathrm{R}$ wave amplitude in leads I, aVL, V5 and V6, S wave amplitude in leads III, aVL, V1, V2, V3.

Based on the measures we used in our study selected ECG criteria for LVH detection in cases with LBBB: QRS > $160 \mathrm{~ms}, \mathrm{SV} 1 \geq 30 \mathrm{~mm}, \mathrm{R}$ V5 or V6 $>25 \mathrm{~mm}$, SV1+RV5 or V6 $\geq 45 \mathrm{~mm}$, Cornell index RaVL+SV3 $>20 \mathrm{~mm}$ (women), $28 \mathrm{~mm}$ (men), $\mathrm{RaVL} \geq 7.5 \mathrm{~mm}, \mathrm{RI}+\mathrm{SIII} \geq 25 \mathrm{~mm}$ and presence of left atrial enlargement $-\mathrm{P}$ wave duration in II $>110 \mathrm{~ms}$ and/or $\mathrm{P}$ terminal force in $\mathrm{V} 1 \geq 0.04 \mathrm{mV} / \mathrm{s}$.

\section{CMRI and left ventricular mass measurement}

Cardiac magnetic resonance was performed with the use of a 1.5 Tesla scaner (Magnetom Avanto, Siemens, Erlangen, Germany). Scout images followed by 2-chamber and 4-chamber long-axis images of the LV with the use of a steady state free precession were obtained to find the final short axis imaging planes. Short-axis images were registered from the mitral valve insertion points to apex with 8 to 10 slices to encompass the entire LV. The imaging parameters were as follows: repetition time of $30 \mathrm{~ms}$, echo time of $1.15 \mathrm{~ms}$, flip angle of $80^{\circ}$, matrix of $156 \times 192$, field of view $276 \times 340 \mathrm{~mm}$, slice thickness of $8 \mathrm{~mm}$ with slice gap of $1.6 \mathrm{~mm}$ and temporal resolution $<30$ ms. Images were analyzed using dedicated software (MASS, Medis, Leiden, Netherlands). Initially, short axis images were previewed from base to apex in a cinematic mode, then endocardial and epicardial contours for end-diastole and end-systole were manually traced and semiautomatically propagated to other frames with subsequent manual adjustments. Delineated contours were used for the quantification of $\mathrm{LV}$ mass and LVMI (LV mass/body surface area). LVH was defined as LVMI exceeding reference values for sex and age in normal population [3].

\section{Statistical analysis}

Continuous variables were reported as the mean \pm standard deviation, categorical variables were expressed as frequency. We used standard unpaired Student $t$ test to compare continuous variables and Pearson X2 test for the comparison of categorical values. Correlations between LVMI and ECG parameters were analyzed using Pearson correlation test. Sensitivity, specificity and overall accuracy of selected ECG criteria were calculated. Receiver operating characteristic (ROC) analysis was also computed to verify diagnostic value of ECG-LVH criteria in relation to CMRI diagnosis. Besides basically implemented ECG-LVH criteria we selected ECG variables of the highest statistical value to compute a combinations to achieve optimal sensitivity and specificity. A p value below 0.05 was considered statistically significant. For the statistical analysis we used SPSS software v. 8.0.

\section{Results}

Mean QRS duration was 164 (134-220) ms, in $34 / 36$ patients $\mathrm{QRS}$ was $\geq 140 \mathrm{~ms}$.

\section{Comparison of patients with and without LVH in CMRI}

According to the CMRI criteria LVH was diagnosed in 17 (47\%) patients (6 women and 11 men). In Table 1 we present the comparison of ECG parameters in two groups - with and without LVH. 
Table 1. Comparison of ECG parameters between two groups with and without left ventricular hypertrophy $(\mathrm{LVH})$ detected in cardiac magnetic resonance imaging.

\begin{tabular}{lcccc}
\hline Parameter & All patients & LVH+ (n=17) & LVH- (n = 19) & P \\
\hline Age [years] & $57 \pm 12(33-80)$ & $56 \pm 14$ & $58 \pm 11$ & NS \\
Women/men & $15 / 21$ & $6 / 11$ & $9 / 10$ & NS \\
LVMI [g/m & $87 \pm 27(42-166)$ & $108 \pm 22$ & $67 \pm 14$ & 0.0001 \\
QRS [ms] & $164 \pm 21(134-220)$ & $170 \pm 25$ & $158 \pm 14$ & NS \\
P wave [ms] & $112 \pm 20(72-147)$ & $119 \pm 23$ & $106 \pm 15$ & 0.05 \\
LA enlargement & $10 / 34(2$ pts AF) & $9 / 17(53 \%)$ & $1 / 17(6 \%)$ & 0.003 \\
R in I [mm] & $7.7 \pm 4.2(1-20)$ & $8.4 \pm 5.2$ & $7.1 \pm 3.1$ & NS \\
S in III [mm] & $8.6 \pm 6.7(0-38)$ & $9 \pm 8.9$ & $8.2 \pm 4.1$ & NS \\
R in aVL [mm] & $7.9 \pm 5.7(2-35)$ & $8.5 \pm 7.7$ & $7.3 \pm 2.9$ & NS \\
S in V1 [mm] & $19.4 \pm 9.4(5-54)$ & $23.2 \pm 10.9$ & $16 \pm 6.2$ & 0.02 \\
S in V2 [mm] & $27.4 \pm 10.1(11-51)$ & $33.6 \pm 8.4$ & $21.8 \pm 8.1$ & 0.0001 \\
S in V3 [mm] & $27.6 \pm 11.7(6-50)$ & $34.6 \pm 10.3$ & $21.3 \pm 9.2$ & 0.0001 \\
R in V5 [mm] & $9.6 \pm 12.3(0-50)$ & $11.2 \pm 13.7$ & $8 \pm 11$ & NS \\
R in V6 [mm] & $12.1 \pm 10.7(3-50)$ & $15 \pm 11.7$ & $9.4 \pm 9.2$ & 0.02 \\
RI+SIII [mm] & $16.3 \pm 9.7(4-58)$ & $17.4 \pm 12.9$ & $15.4 \pm 5.9$ & NS \\
RaVL+SV3 [mm] & $35 \pm 12(15-55)$ & $43 \pm 10$ & $28.6 \pm 9.4$ & 0.0001 \\
SV1+RV5, 6 [mm] & $31 \pm 19(10-104)$ & $37 \pm 23$ & $26 \pm 12$ & NS \\
SV2+SV3 [mm] & $55 \pm 19(17-95)$ & $68 \pm 15$ & $43 \pm 14$ & 0.0001 \\
SV2+RV5, 6 [mm] & $40.2 \pm 16.4(16-89)$ & $49.3 \pm 15.2$ & $31.9 \pm 12.9$ & 0.001 \\
SV2, 3+RV5, 6 [mm] & $44.4 \pm 15.9(16-89)$ & $54.4 \pm 12.7$ & $35.5 \pm 13.1$ & 0.0001 \\
\hline
\end{tabular}

LVMI — left ventricular mass index; LA — left atrial; AF — atrial fibrillation

The most prominent differences were observed in the amplitude of S waves in V2 and V3. Only in V6 the $\mathrm{R}$ wave amplitude was significantly higher in LVH group. Finally we analyzed three additional parameters based on the amplitude of $\mathrm{R}$ and/or $\mathrm{S}$ waves $-\mathrm{S} 2+\mathrm{S} 3, \mathrm{SV} 2+\mathrm{RV} 5$ or $\mathrm{V} 6, \mathrm{SV} 2$ or SV3 + RV5 or RV6.

\section{Correlations of LVMI and ECG parameters}

We correlated LVMI with ECG parameters with following results - QRS: $r=0.2(\mathrm{NS}), \mathrm{P}$ wave duration: $r=0.47(p=0.005)$, RI: $r=0.42(p=0.01)$, SIII: $\mathrm{r}=0.27$ (NS), SV1: $\mathrm{r}=0.53(\mathrm{p}=0.001), \mathrm{SV} 2$ : $r=0.31(\mathrm{NS})$, SV3: $r=0.42(\mathrm{p}=0.01)$, RV5: $r=0.5$ $(\mathrm{p}=0.002)$, RV6: $\mathrm{r}=0.61(\mathrm{p}=0.0001), \mathrm{RI}+\mathrm{SIII}:$ $\mathrm{r}=0.37(\mathrm{NS}), \mathrm{SV} 1+\mathrm{RV} 5,6: \mathrm{r}=0.64(\mathrm{p}=0.001)$, RaVL+SV3: $r=0.5(p=0.002)$. Correlations with additional parameters were as follows: SV2+SV3: $\mathrm{r}=0.42(\mathrm{p}=0.01), \mathrm{SV} 2+\mathrm{RV} 5,6: \mathrm{r}=0.71(\mathrm{p}=0.0001)$, SV2, 3+RV5, 6: $r=0.75(\mathrm{p}=0.0001)$ (Fig. 1).

\section{Predictive value of ECG for $\mathrm{LVH}$ in $\mathrm{LBBB}$}

Results of the ROC analysis are shown in Table 2. The highest values (area under curve $>0.8$ ) were noted for parameters that included $\mathrm{S}$ wave amplitude: $\mathrm{S}$ in V2, V3 i.e. RaVL+SV3, SV2+SV3,

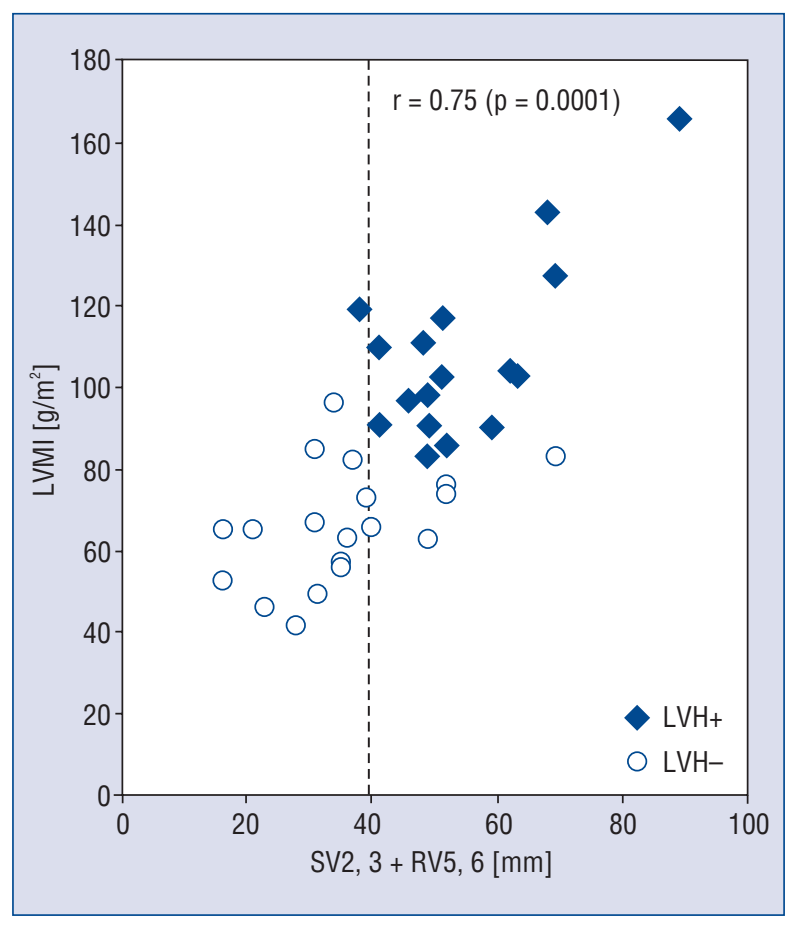

Figure 1. Correlation between left ventricular mass index (LVMI) and maximal sum of amplitude of $S$ wave in V2 or V3 and R wave in V5 or V6; LVH - left ventricular hypertrophy. 
Table 2. ROC - analysis for ECG-left ventricular hypertrophy criteria.

\begin{tabular}{lcc}
\hline Parameter & Area under curve & P \\
\hline QRS duration & 0.635 & NS \\
P wave duration & 0.687 & NS \\
R aVL & 0.497 & NS \\
SV1 & 0.711 & 0.03 \\
SV2 & 0.848 & 0.0001 \\
SV3 & 0.837 & 0.0001 \\
RaVL+SV3 & 0.875 & 0.0001 \\
RV6 & 0.728 & 0.02 \\
R I+SIII & 0.491 & NS \\
SV1+RV5, 6 & 0.655 & NS \\
SV2+SV3 & 0.882 & 0.0001 \\
SV2+RV5, 6 & 0.856 & 0.0001 \\
SV2, 3+RV5, 6 & 0.861 & 0.0001 \\
\hline
\end{tabular}

SV2+RV5 or V6, SV2 or V3+RV5 or V6. Based on the ROC analysis we proposed the new cut-off values for: Cornell index (37 mm), SV2+SV3 $(60 \mathrm{~mm})$, $\mathrm{SV} 2+\mathrm{RV} 5$ or V6 $(40 \mathrm{~mm})$ and $\mathrm{SV} 2$ or $\mathrm{V} 3+\mathrm{RV} 5$ or V6 $(40 \mathrm{~mm})$. In Table 3 we present the sensitivity, specificity and overall accuracy of the ECG criteria for LVH detection in presence of LBBB. The highest clinical value was observed for the ECG parameters that included $\mathrm{S}$ wave amplitude in V2 and/or V3.

\section{Discussion}

It is well known that patients with LBBB have more frequently hypertrophied LV than those with QRS without intraventricular conduction abnormalities [8]. Several studies correlated ECG LVH parameters in patients with $\mathrm{LBBB}$ with the results of autopsy or echocardiographic study [7-14]. The most frequently cited ECG criterion for $\mathrm{LVH}$ in patients with LBBB (S in V2+RV6 > $45 \mathrm{~mm}$ ) was reported with maximal $86 \%$ sensitivity and $100 \%$ specificity [15].

Now the results of CMRI are more accurate and reproducible in LV mass quantification. CMRI is also used to determine the reason of LVH [16]. In some studies data from CMRI was compared with ECG measurements, to our knowledge there was no study that did it for patients with LBBB.

Our study presented that in patients with LBBB and CMRI defined LVH the most prominent differences in ECG parameters between $\mathrm{LVH}+$ and LVH- groups were observed in S wave amplitude in $\mathrm{V} 2$ and $\mathrm{V} 3$ and in the parameters that used $\mathrm{S}$ wave amplitude (V2 and/or V3). The highest correlation coefficients for ECG parameters and LVMI were observed for combination of the amplitude of $\mathrm{S}$ waves measured in $\mathrm{V} 2$ or $\mathrm{V} 3$ and $\mathrm{R}$ waves amplitude measured in $\mathrm{V} 5$ or $\mathrm{V} 6-\mathrm{r}=0.71$ and $\mathrm{r}=0.75$. In one study that correlated LVMI calculated with CMRI and ECG parameters in the group of patients with aortic stenosis, correlation coefficients between ECG criteria and LVMI were comparable to those in our LBBB group: Cornel index: 0.47 (LBBB: $r=0.5)$, SV1+RV5, 6: 0.57 (LBBB: $r=$ $=0.64)$, RI+SIII: 0.33 (LBBB: $\mathrm{r}=0.37)[16]$. However in the ROC analysis the results were slightly different (Table 4).

In the Table 4 we compared the results of ROC analysis, sensitivity and specificity of the parameters that were used in three ECG/CMRI studies [17, 18]. The compared populations and cut-off ECG points were different (general multiethnic population in MESA study, aortic valve disease patients in the second and our LBBB group). LBBB patients

Table 3. Sensitivity, specificity and overall accuracy of selected ECG parameters in left ventricular hypertrophy diagnosis.

\begin{tabular}{lccc}
\hline Parameter & Sensitivity & Specificity & Overall accuracy \\
\hline QRS $>160 \mathrm{~ms}$ & $65 \%$ & $58 \%$ & $61 \%$ \\
Left atrial enlargement & $53 \%$ & $94 \%$ & $74 \%$ \\
$\mathrm{RaVL} \geq 7.5 \mathrm{~mm}$ & $47 \%$ & $57 \%$ & $53 \%$ \\
$\mathrm{RI}+\mathrm{SIII} \geq 25 \mathrm{~mm}$ & $18 \%$ & $95 \%$ & $58 \%$ \\
$\mathrm{~S}$ in V1 $\geq 30 \mathrm{~mm}$ & $18 \%$ & $95 \%$ & $58 \%$ \\
$\mathrm{R}$ V5or RV6 $>25 \mathrm{~mm}$ & $12 \%$ & $95 \%$ & $55 \%$ \\
SV1+RV5, $>35 \mathrm{~mm}$ & $35 \%$ & $79 \%$ & $58 \%$ \\
SV2+SV3 $>60 \mathrm{~mm}$ & $88 \%$ & $89 \%$ & $89 \%$ \\
SV2+RV5, $2 \geq 40 \mathrm{~mm}$ & $76 \%$ & $79 \%$ & $78 \%$ \\
SV2, 3+RV5, $>40 \mathrm{~mm}$ & $94 \%$ & $79 \%$ & $86 \%$ \\
RaVL+SV3 $>37 \mathrm{~mm}$ & $88 \%$ & $89 \%$ & $89 \%$ \\
\hline
\end{tabular}


Table 4. Comparison of ROC analysis, sensitivity and specificity of selected parameters in three cardiac magnetic resonance imaging studies.

\begin{tabular}{|c|c|c|c|c|c|c|c|c|c|}
\hline & \multicolumn{3}{|c|}{$\begin{array}{l}\text { MESA study } \\
\text { (n = 4967) }\end{array}$} & \multicolumn{3}{|c|}{$\begin{array}{l}\text { Aortic valve disease } \\
\qquad(\mathrm{n}=120)\end{array}$} & \multicolumn{3}{|c|}{ Our study } \\
\hline & ROC & Sensitivity & Specificity & ROC & Sensitivity & Specificity & ROC & Sensitivity & Specificity \\
\hline $\mathrm{RaVL}+\mathrm{SV} 3$ & 0.56 & $15.1 \%$ & $97.3 \%$ & 0.78 & $52 \%$ & $87 \%$ & 0.87 & $88 \%$ & $89 \%$ \\
\hline SV1+RV5, V6 & 0.59 & $26.0 \%$ & $92.6 \%$ & 0.86 & $52 \%$ & $87 \%$ & 0.65 & $35 \%$ & $79 \%$ \\
\hline
\end{tabular}

MESA and aortic valve disease study used the following cut-points: for RaVL+SV3 $>2.8 \mathrm{mV}$ in men and $>2.0 \mathrm{mV}$ in women; for $\mathrm{SV} 1+\mathrm{RV} 5, \mathrm{~V} 6 \geq 3.5 \mathrm{mV}$

were excluded in the aortic valve study; there is no data about LBBB patients in MESA study. The best ECG results in the prediction of LVH were recorded in our small LBBB study. We know that such small number of analyzed cases decreased the value of our results but our findings were achieved with recalibrated parameters. It is not a new conception. The necessity of recalibration ECG LVH criteria (CMRI as a reference) in patients with QRS $<120 \mathrm{~ms}$ was postulated by Alfakih et al. [19]. They proposed new cut-off values for Sokolov and Cornell indexes separately for male and female patients. It should be also pointed that the frequency of the presence of the LVH in analyzed population may be an important factor that determines the sensitivity/specificity results. LVH is much more frequently observed in patients with LBBB. Havelda et al. [13] in their study from 1982 observed higher accuracy of the ECG for LVH detection in patients with more pronounced LVH. Opposite observation reported Kafka et al. [14] in 1985 in echocardiography/ECG study of 100 patients with LBBB. The sensitivity and specificity was lower in cases with LVMI > $215 \mathrm{~g} / \mathrm{m}^{2}$ than in those with LVMI $>115 \mathrm{~g} / \mathrm{m}^{2}$. LVH ECG parameters were also verified with computer tomography in the study conducted by Truong et al. [20]. The Cornel and Sokolov index achieved $22 \%$ and $35 \%$ sensitivity and $97 \%$ and $90 \%$ specificity. The authors did not postulate the necessity of recalibration. LVH ECG parameters were also correlated with the autopsy results. Zmyslinski et al. [11] correlated LV mass with selected ECG parameters. The maximum value for correlation coefficient achieved 0.375 for $\mathrm{R}$ wave amplitude in aVL and 0.373 for SV1 or SV2+RV5 or RV6. In our study we used LVMI and $r$ values were much higher - up 0.75 . These values were lower for non-indexed LV mass. What is interesting, the mean heart weight of patients with complete LBBB in this study was similar to mean LV mass in our study. The analyzed populations in those 2 studies are different and cannot be simply compared. Peterson and Tikoff [10] in their clinical-pathologic study of 50 patients with LBBB postulated that LVH could not be predicted with ECG criteria. However as authors commented the association of LVH and LBBB was probably less pronounced in living patients. In 1984 Klein et al. [15] based on the echocardiographic verification proposed the following parameter sum of the $\mathrm{S}$ amplitude in $\mathrm{V} 2$ and $\mathrm{R}$ wave amplitude in $\mathrm{V} 6$ above $45 \mathrm{~mm}$. The reported sensitivity achieved $86 \%$, specificity $100 \%$. Additionally LVH diagnosis was supported by the presence of left atrial enlargement and QRS duration > $160 \mathrm{~ms}$. Important value of the presence of the left atrial enlargement in LVH detection was also reported in our study. Kafka et al. [14] in echocardiography/ECG study reported comparable results in 100 patients with LBBB. In our study the highest accuracy for LVH detection in LBBB ECG was achieved when the combination of the amplitude of $\mathrm{S}$ waves in $\mathrm{V} 2$ $-\mathrm{V} 3$ and $\mathrm{R}$ waves in aVL or V5, V6. Similar observation reported Zmyslinski et al. [11] however it was defined as maximal sum of $\mathrm{R}$ and $\mathrm{S}$ wave in any precordial lead.

ECG is not a perfect tool in the LV mass estimation and definition of presence/absence of the LVH. However ECG presents information on the cardioelectric field in hypertrophied hearts and this information (as it is pointed by Bacharova [21]) is an added value of ECG. This can possibly have important impact on the value of ECG as a predictor of cardiac events.

Additional comment is necessary to our results of patients with hypertrophic cardiomyopathy. There were 4 of them with LBBB and QRS duration/LVMI as follows: $170 \mathrm{~ms} / 104 \mathrm{~g} / \mathrm{m}^{2}, 190 \mathrm{~ms} /$ $/ 166 \mathrm{~g} / \mathrm{m}^{2}, 156 \mathrm{~ms} / 53 \mathrm{~g} / \mathrm{m}^{2}$ (no LVH criteria in CMRI), $140 \mathrm{~ms} / 143 \mathrm{~g} / \mathrm{m}^{2}$. Generally in our group and in hypertrophic cardiomyopathy patients there was no relation between QRS duration and LV mass in CMRI. The cases with hypertrophic cardiomyopathy were selected for the study based of previously declared LBBB criteria. On the Figure 2 there is an example of ECG of the first reported case with hypertrophic cardiomyopathy. 


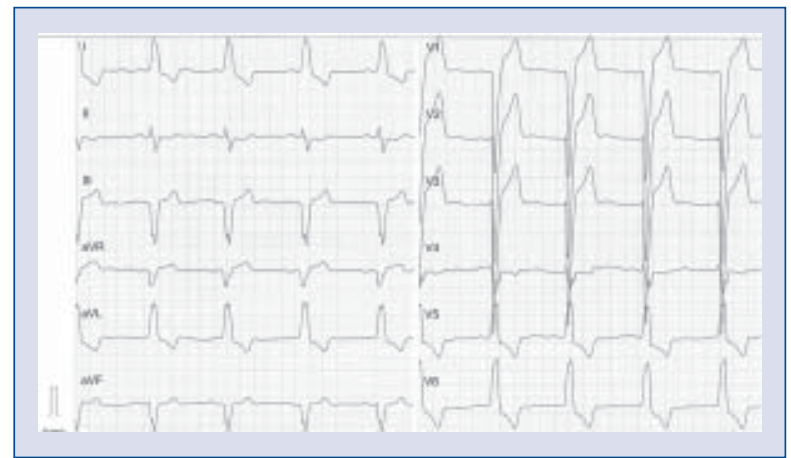

Figure 2. ECG of 56 years men with hypertrophic cardiomyopathy; QRS $170 \mathrm{~ms}$, left ventricular mass index $104 \mathrm{~g} / \mathrm{m}^{2}$.

\section{Limitations of the study}

Our study has limitations that merit discussion. The most important limitation is the small number of patients included in this study. Our population is inhomogeneous. We did not analyze consecutive patients with LBBB, we analyzed only those that had CMRI. Therefore our population is probably not fully typical with clinical profile of patients with LBBB. As we could observe in literature the number of patients with LBBB analyzed in previous studies was higher but none of them used CMRI as a reference for LVH detection. We also could not analyze the gender and race differences. We were unable to verify proposed criteria using new set of patients.

The assessment of LV measurements by MRI has limitations. In general they are related to the partial volume effects and the problem whether or not include the most basal slice of the MRI into the measurements of LV volumes. This can results in $\pm 10 \%$ variations in measured volumes.

\section{Conclusions}

In patients with LBBB the ECG parameters based on the amplitude of $\mathrm{S}$ wave in $\mathrm{V} 2$ or $\mathrm{V} 3$ and $\mathrm{R}$ wave in the leads aVL, V5, V6 have the highest clinical value in the prediction of the LVH determined by CMRI. It was necessary to recalibrate these parameters and it is needed to verify them in larger number of LBBB patients.

\section{Acknowledgements}

The study was financed by statue program of the Institute of Cardiology.

Conflict of interest: none declared

\section{References}

1. Surawicz B, Knilans TK. Chou's electrocardiography in clinical practice. W.B. Saunders Company, Philadelphia 2001.

2. Lev M, Unger PN, Rosen KM, Bharati S. The anatomic substrate of complete left bundle branch block. Circulation, 1974; 50: 479-486.

3. Maceira AM, Prasad SK, Khan M, Pennel DJ. Normalized left ventricular sytolic and diastolic function by steady state free precession cardiovascular magnetic resonance. J Cardiovasc Magn Reson, 2006; 8: 417-426.

4. Sugeng L, Mor-Avi V, Weinert L et al. Quantitative assessment of left ventricular size and function: Side-by-side comparison of real-time three-dimensional echocardiography and computed tomography with magnetic resonance reference. Circulation, 2006; 114: 654-661.

5. Pouleur AC, Polain de Waroux JB, Pasquet A et al. Assessment of left ventricular mass and volumes by three-dimensional echocardiography in patients with or without wall motion abnormalities: Comparison against cine magnetic resonance imaging. Heart, 2008; 94: 1050-1057.

6. Bottini PB, Carr AA, Prisant LM, Flickinger FW, Allison JD, Gottdiener JS. Magnetic resonance imaging compared to echocardiography to assess left ventricular mass in the hypertensive patient. Am J Hypertens, 1995; 8: 221-228.

7. Surawicz B, Childers R, Deal BJ, Gettes LS AHA/ACCF/HRS Recommendations for the Standardization and Interpretation of the Electrocardiogram. Part III: Intraventricular Conduction Disturbances. J Am Coll Cardiol, 2009; 53: 976-981.

8. Scott RC, Norris RJ Electrocardiographic-pathologic correlation study of left ventricular hypertrophy in the presence of left bundle branch block. Circulation, 1959; 20: 766.

9. Cokkinos DV, Demopoulos JN, Heimonas ET, Mallios C, Papazoglou N, Vorides EM. Electrocardiographic criteria of left ventricular hypertrophy in left bundle branch block. Br Heart J, 1978; 40: 320-324.

10. Petersen RV, Tikoff G. Left bundle branch block and left ventricular hypertrophy: Electrocardiographic-pathologic correlations. Chest, 1971; 59: 174-177.

11. Zmyslinski RW, Richeson JF, Akiyama T. Left ventricular hypertrophy in presence of complete left bundle branch block. Br Heart J 1980; 43: 170-175.

12. Vandenberg BF, Romhilt DW. Electrocardiographic diagnosis of left ventricular hypertrophy in the presence of bundle branch block. Am Heart J, 1991; 122: 818-822.

13. Havelda CJ, Sohi GS, Flowers NC, Horan LG. The pathologic correlates of the electrocardiogram: Complete left bundle branch block. Circulation, 1982; 65: 445-451.

14. Kafka H, Burggraf GW, Milliken JA. Electrocardiographic diagnosis of left ventricular hypertrophy in the presence of left bundle branch block: An echocardiographic study. Am J Cardiol, 1985; 55: 103-106.

15. Klein RC, Vera Z, DeMaria AN, Mason DT. Electrocardiographic diagnosis of left ventricular hypertrophy in the presence of left bundle branch block. Am Heart J, 1984; 108 (3 Part 1): 502-506.

16. Germans T, Nijveldt R, Brouwer WP et al. The role of cardiac magnetic resonance imaging in differentiating the underlying causes of left ventricular hypertrophy. Neth Heart J, 2010; 18: 135-143.

17. Buchner S, Debl K, Haimerl J et al. Electrocardiographic diagnosis of left ventricular hypertrophy in aortic valve disease: Evaluation of ECG criteria by cardiovascular magnetic resonance. J Cardiovasc Magn Reson, 2009; 11: 18.

18. Jain A, Tandri H, Dalal D et al. Diagnostic and prognostic utility of electrocardiography for left ventricular hypertrophy defined by magnetic resonance imaging in relationship to ethnicity: The Multi-Ethnic Study of Atherosclerosis (MESA). Am Heart J, 2010; 159: 652-658.

19. Alfakih K, Walters K, Jones T, Ridgway J, Hall AS, Sivananthan M. New gender-specific partition values for ECG criteria of left ventricular hypertrophy: recalibration against cardiac MRI. Hypertension, 2004; 44: 175-179.

20. Truong QA, Ptaszek LM, Charipar EM et al. Performance of electrocardiographic criteria for left ventricular hypertrophy as compared with cardiac computed tomography: From the Rule Out Myocardial Infarction Using Computer Assisted Tomography trial. J Hypertens, 2012; 28: 1959-1967.

21. Bacharova L. Electrocardiography: Left ventricular mass discrepancies in left ventricular hypertrophy: Electrocardiography imperfection or beyond perfection? J Electrocardiography, 2009; 42: 593-596. 